\title{
Video Article \\ Minimally Invasive Embryo Transfer and Embryo Vitrification at the Optimal Embryo Stage in Rabbit Model
}

\author{
Ximo Garcia-Dominguez ${ }^{1}$, Francisco Marco-Jiménez ${ }^{1}$, Maria Pilar Viudes-de-Castro ${ }^{1}$, José Salvador Vicente ${ }^{1}$ \\ ${ }^{1}$ Departamento de Ciencia Animal, Universitat Politècnica de Valencia
}

Correspondence to: José Salvador Vicente at jjvicent@dca.upv.es

URL: https://www.jove.com/video/58055

DOI: doi:10.3791/58055

Keywords: Embryo, embryo transfer, laparoscopy, vitrification, cryopreservation, rabbit

Date Published: 3/13/2019

Citation: Garcia-Dominguez, X., Marco-Jiménez, F., Viudes-de-Castro, M.P., Vicente, J.S. Minimally Invasive Embryo Transfer and Embryo Vitrification at the Optimal Embryo Stage in Rabbit Model. J. Vis. Exp. (), e58055, doi:10.3791/58055 (2019).

\section{Abstract}

Assisted reproductive techniques (ARTs), such as in vitro embryo culture or embryo cryopreservation, affect natural development patterns with perinatal and postnatal consequences. To ensure the innocuousness of ART applications, studies in animal models are necessary. In addition, as a last step, embryo development studies require evaluation of their capacity to develop full-term healthy offspring. Here, embryo transfer to the uterus is indispensable to perform any ARTs-related experiment.

The rabbit has been used as a model organism to study mammalian reproduction for over a century. In addition to its phylogenetic proximity to the human species and its small size and low maintenance cost, it has important reproductive characteristics such as induced ovulation, a chronology of early embryonic development similar to humans and a short gestation that allow us to study the consequences of ART application easily. Moreover, ARTs (such as intracytoplasmic sperm injection, embryo culture, or cryopreservation) are applied with suitable efficiency in this species.

Using the laparoscopic embryo transfer technique and the cryopreservation protocol presented in this article, we describe 1) how to transfer embryos through an easy, minimally invasive technique and 2) an effective protocol for long-term storage of rabbit embryos to provide timeflexible logistical capacities and the ability to transport the sample. The outcomes obtained after transferring rabbit embryos at different developmental stages indicate that morula is the ideal stage for rabbit embryo recovery and transfer. Thus, an oviductal embryo transfer is required, justifying the surgical procedure. Furthermore, rabbit morulae are successfully vitrified and laparoscopically transferred, proving the effectiveness of the described techniques.

\section{Video Link}

The video component of this article can be found at https://www.jove.com/video/58055/

\section{Introduction}

With the aims of bypassing human infertility or improving dissemination of livestock of high genetic value and preserving animal genetic resources, a set of techniques collectively termed assisted reproduction technologies, such as superovulation, in vitro fertilization, embryo culture, or cryopreservation, were developed ${ }^{1,2}$. Currently, hormonal treatments are given to stimulate the ovaries and produce a large number of antral ovarian follicles ${ }^{1}$. Oocytes collected from these follicles can be matured, fertilized, and developed in vitro until they are either cryopreserved or transferred to surrogate mothers ${ }^{3}$. However, during these treatments, gametes and zygotes are exposed to a series of nonphysiological processes that could require embryo adaptation to survive in these conditions $s^{4,5}$. This adaptation is possible due to early embryo plasticity, which allows embryo changes in gene expression and developmental programming ${ }^{6}$. However, these modifications can influence the subsequent stages of embryo development until adulthood, and it is now widely accepted that methods, timing, cryopreservation procedure or culture conditions show different outcomes on embryo fate ${ }^{7,8}$. Therefore, to elucidate the specific induced effects of ARTs, the use of wellcharacterized animal models is inevitable.

The first documented live birth resulting from transfer of mammalian embryos took place in $1890^{9}$. Today, embryo transfer (ET) to a surrogate female is a crucial step in studying the ART-induced effects during preimplantation on subsequent embryo development stages ${ }^{10}$. ET techniques depend on the size and anatomical structure of each animal. In the case of large-sized animal models, it has been possible to perform ET by transcervical nonsurgical ET techniques, but in smaller-size species catheterization of the cervix is more complex and surgical techniques are frequently used ${ }^{11}$. However, surgical ET can cause hemorrhaging that could impair implantation and embryo development, as blood can invade the uterine lumen, causing embryo death ${ }^{10}$. Transcervical nonsurgical ET techniques are still applied in humans, baboons, bovine, pigs and mice $e^{12,13,14,15,16,17}$, but surgical ETs are still being used in species such as goats, sheep or other animals which present additional difficulties $^{10,18,19,20,21}$, such as rabbits (two independent cervices) or mice (small size). Nonetheless, surgical transfer methods tend to have gradually been replaced by less invasive methods. Endoscopy was used to transfer embryos, for example, in rabbits, pigs and small ruminants ${ }^{18,19,20}$. These minimally invasive endoscopy methods can be used to transfer embryos into the ampulla via the infundibulum, which is essential in rabbits and has demonstrated beneficial effects in some species ${ }^{20}$. This is based on the importance of the correct dialogue between 
embryo and mother during early embryo stages in the oviduct. As mentioned above, the embryo remodeling that takes place in rabbits during embryo migration through the oviduct is essential to achieve embryos able to implant ${ }^{22,23}$.

Larger-size animal models, such as bovine, are interesting because the biochemical and preimplantation features are similar to those in human species $^{24}$. However, large animals are too expensive to use in preliminary trials, and rodents are considered an ideal model $(76 \%$ model organisms are rodents) for laboratory research ${ }^{25}$. Nevertheless, the rabbit model provides some advantages over rodents in reproductive studies, as some reproductive biological processes exhibited by humans are more similar in rabbits than those in mice. Human and rabbits present a similar chronological embryonic genome activation, gastrulation and hemochorial placenta structure. In addition, using rabbits it is possible to know the exact timing of fertilization and pregnancy stages due to their induced ovulation ${ }^{25}$. Rabbit life cycles are short, completing gestation in 31 days and reaching puberty at about 4-5 months; the animal is easy to handle due to its docile and non-aggressive behavior, and its upkeep is very economical compared to the expense of larger animals. Moreover, it is crucial to mention that rabbits have a duplex uterus with two independent cervixes ${ }^{11,25}$. This places the rabbit in a preferential position, as embryos from the different experimental groups can be transferred into the same animal, but into a different uterine horn. This allows us to compare both experimental effects, reducing the maternal factor from the results.

Today, nonsurgical ET methods are not in use in rabbit. Some studies carried out in the late $90 \mathrm{~s}$ using a transcervical ET technique resulted in low delivery rates ranging from $5.5 \%$ to $20.0 \%{ }^{11,26}$ versus $50-65 \%$ by surgical methods, among them the laparoscopy procedure described by Besenfelder and Brem ${ }^{18}$. The low success rates of these nonsurgical ET methods in rabbits coincide with the lack of the necessary embryo remodeling in the oviduct, which is avoided in transcervical ET. Here, we describe an effective minimally invasive laparoscopic ET procedure using rabbits as a model organism. This technique provides a model for further reproductive research in large animals and humans.

Because rabbits have a particularly narrow time window for embryo implantation, ET in this species requires a high degree of synchrony between the developmental stage of the embryo at ET and the physiological status of the recipient ${ }^{27}$. In some cases, after a reproductive treatment that slows embryo development (such as in vitro culture) or alters the endometrial receptivity (such as superovulation treatments), there is no synchrony between the embryo and the maternal uterus. These situations can negatively affect outcome. To respond in these contexts, we describe an effective rabbit morula vitrification protocol that allows us to pause, organize and resume the experiments. This process is logistically desirable for reproductive studies and gives us the capacity for long-term storage of embryos, allowing their transport. The laparoscopic procedure and cryopreservation strategies allow better planning of studies with fewer animals. Thus, our methodology offers hygienic and economic advantages and conforms to the concept of the 3Rs (replacement, reduction and refinement) of animal research with the stated goal of improving human treatment of experimental animals. Thus, with these methods, rabbits constitute an ideal model organism for in vivo reproductive assays.

\section{Protocol}

All experimental procedures used in this study were performed in accordance with Directive 2010/63/EU EEC for animal experiments and reviewed and approved by the Ethical Committee for Experimentation with Animals of the Polytechnic University of Valencia, Spain (research code: 2015/VSC/PEA/00170).

\section{Embryo Transfer}

\section{Preparation of recipient females}

1. Use only sexually mature females (> 4.5 months old).

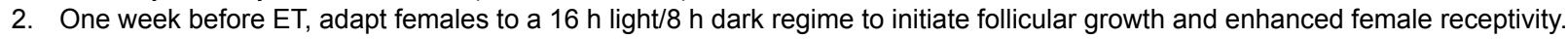

3. Select the recipient females, observing the turgidity and color of the vulva. If the vulva is turgid and reddish, the female is receptive.

4. Induce pseudopregnancy (ovulation) by a single intramuscular injection of $1 \mu \mathrm{g}$ of buserelin acetate (synthetic analogue of Gonadotropin-releasing hormone) regardless of body weight.

NOTE: Normally, $0.8 \mu \mathrm{g}$ is a suitable dose for ovulation induction in medium-size rabbits $(4-5 \mathrm{~kg})$, so $1 \mu \mathrm{g}$ generally guarantees the ovulation.

5. Induce ovulation as many days beforehand as the age of the embryos to be transferred (for example, $70-72 \mathrm{~h}$ before fresh morula ET).

\section{Anesthesia and analgesia}

1. Weigh the rabbit and load the following anesthetics and analgesics

1. In a $1 \mathrm{~mL}$ syringe with a $30 \mathrm{G}$ needle: load xylazine $(5 \mathrm{mg} / \mathrm{kg})$ and buprenorphine hydrochloride $(0.03 \mathrm{mg} / \mathrm{kg})$. In another $1 \mathrm{~mL}$ syringe with a $23 \mathrm{G}$ pericranial needle, load ketamine hydrochloride $(35 \mathrm{mg} / \mathrm{kg})$.

2. Hold the rabbit and inject the xylazine-buprenorphine mixture intramuscularly.

3. Insert the pericranial needle with ketamine in the marginal ear vein, slowly introducing all the syringe contents intravenously.

4. Fix the needle and leave it inserted throughout the remaining steps to administer more anesthesia if necessary.

5. Leave the rabbit in the cage (clean and without any other animals) on a warm stage.

6. Once unconscious, apply eye ointment to avoid dryness of the eye and check for the absence of the palpebral freflex. NOTE: This protocol provides a surgical anesthesia plane for a minimum of $30 \mathrm{~min}$. If a longer time is required, inject additional dosages with half of the amount of ketamine hydrochloride described in 1.2.1 after $30 \mathrm{~min}$.

7. Monitor the depth of anesthesia by checking the pedal reflex and breathing movement. Changes in the breathing pattern to an irregular and faster rate indicate loss of the proper plane of anesthesia.

8. Monitor the color of the mucous membranes (eyes, lips, etc.), respiratory rate (30-60 breaths per minute), heart rate (120-325 beats per minute) and rectal temperature $\left(38-39.6^{\circ} \mathrm{C}\right)$.

9. Eight hours before transfer, withhold food from animals to avoid the greater gut size and activity until the ET process is finished. Leave free access to water. 


\section{Embryo preparation}

1. Warm the embryo manipulation media to $25^{\circ} \mathrm{C}$ : Base Medium (BM), consisting of Dulbecco's Phosphate-Buffered Saline (DPBS) supplemented with $0.2 \%(\mathrm{w} / \mathrm{v})$ of Bovine Serum Albumin.

2. Working under a stereomicroscope, rinse fresh or thawed (Step 2) embryos with BM.

3. Using sterile gloves, attach an appropriately configured $17 \mathrm{G}$ epidural catheter to a $1 \mathrm{~mL}$ syringe.

4. Aspirate $1 \mathrm{~cm}$ of BM into the catheter, followed by a small air bubble.

5. Aspirate 5-7embryos in a volume of $10 \mu \mathrm{L}$ of BM, followed by another small air bubble.

6. Finish loading the catheter by aspirating $1 \mathrm{~cm}$ of BM.

\section{Embryo transfer}

1. Use sterile gloves; wear a gown and mask.

2. Sterilize surgical instruments, clean the surfaces where surgery will be performed, and wipe them with $70 \%$ ethanol.

3. Perform anesthesia as previously detailed (step 1.2), checking for loss of reflexes.

4. Shave the fur from the ventral abdomen with an electric razor.

5. Prepare the ventral abdomen aseptically.

1. Clean the surgical area and remove any remaining hair. Evacuate the bladder using a urinary catheter. Wash the surgical area with a chlorhexidine gluconate soap. Sanitize the area with chlorhexidine solution.

6. Place the animal on a warm surgical table, in Trendelenburg's position (head down at $45^{\circ}$ ) to ensure that the stomach and intestines are cranially located. If any viscera are damaged in the process, the animal may die. It is therefore important to have them properly located (Figure 1).

7. Cover the area using a sterile towel, with a hole (fenestration) exposing the shaved area, to separate the surgical site from any potential contaminating areas.

8. Insert one endoscopic trocar $5 \mathrm{~cm}$ into the abdominal cavity, $2 \mathrm{~cm}$ caudal to the xiphoid process, and insufflate through it the peritoneal cavity with a pressure-regulating mechanical insufflator.

NOTE: The intra-abdominal pressure should be $8-12 \mathrm{mmHg}$ with $\mathrm{CO}_{2}$ (Figure 1A).

9. Insert the endoscope camera through the endoscopic trocar (Figure 1B).

NOTE: Identify the reproductive tract, determining the status and position of the infundibulum and ampulla before ET to facilitate the next steps.

10. Insert the $17-\mathrm{G}$ epidural needle into the inguinal region between $2-3 \mathrm{~cm}$ from the infundibulum (Figure 1B).

11. Identify the entrance of the infundibulum (Figure 2A, 2B).

12. Insert the loaded catheter (step 1.3) through the epidural needle into the abdomen (Figure 1C).

13. Locate the oviduct and insert $1-2 \mathrm{~cm}$ of the epidural catheter through the infundibulum in the ampulla (Figure 2A-2C). Do not progress very far into the oviduct to prevent damage and hemorrhage.

14. Release the embryos into the oviduct by gently pressing the plunger of the syringe coupled to the catheter (Figure 2D-2F). Both air bubbles must exit the catheter.

15. Remove the catheter just after the embryos have been released.

16. Rinse the catheter, aspirating and releasing manipulating medium to check the absence of the embryos and confirm their successful transfer.

17. Repeat steps 1.4 .11 to 1.4 .16 in the other side of the uterus, if desired.

18. Remove the epidural needle and endoscope camera.

19. Release $\mathrm{CO}_{2}$ through the endoscopic trocar. If excess gas remains in the abdomen of the animal, it will have pain and discomfort.

20. Remove the endoscopic trocar from the abdominal cavity. Remove the surgical towel.

21. Discontinue anesthesia.

22. Cleanse the incision made by the trocar with povidone iodide solution. Close the incision made by the trocar with a plastic dressing.

\section{Postoperative care}

1. Treat the animals with antibiotics: $10 \mathrm{mg} / \mathrm{kg}$ of enrofloxacin, subcutaneously, every $24-\mathrm{h}$ for 5 days.

2. Administer analgesics: buprenorphine hydrochloride $(0.03 \mathrm{mg} / \mathrm{kg})$, intramuscularly, each 12 hours for 3 days; Meloxicam ( $0.2 \mathrm{mg} / \mathrm{kg})$, subcutaneously, every $24-\mathrm{h}$ for 3 days.

3. Monitor the animals for at least 30 min after surgery (depending on the animal and the dose of anesthesia used) making sure they recover their physiological conditions.

4. Identify the recipient (e.g., ear tattoo) and house animals individually in a clean cage with the appropriate environmental condition. 

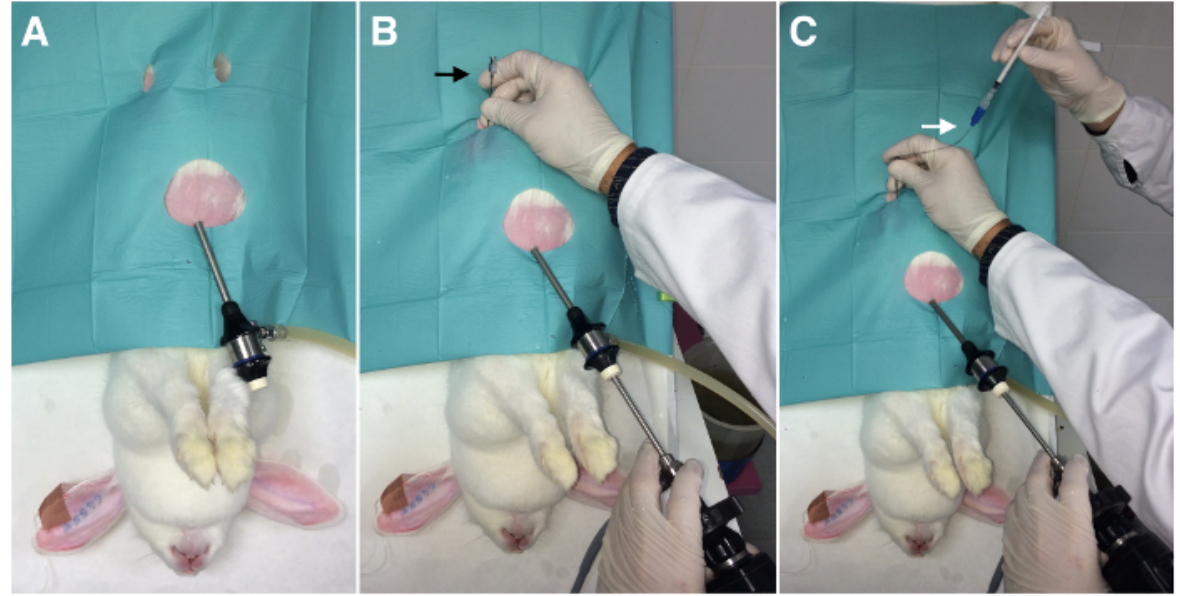

Figure 1: Laparoscopic embryo transfer assisted by laparoscopy (External view). A) Insertion of the endoscopic trocar (one port). B) Insertion of the endoscopic camera and the epidural needle (black arrow). C) Insertion of the embryo transfer catheter (white arrow) through the epidural needle. Please click here to view a larger version of this figure.
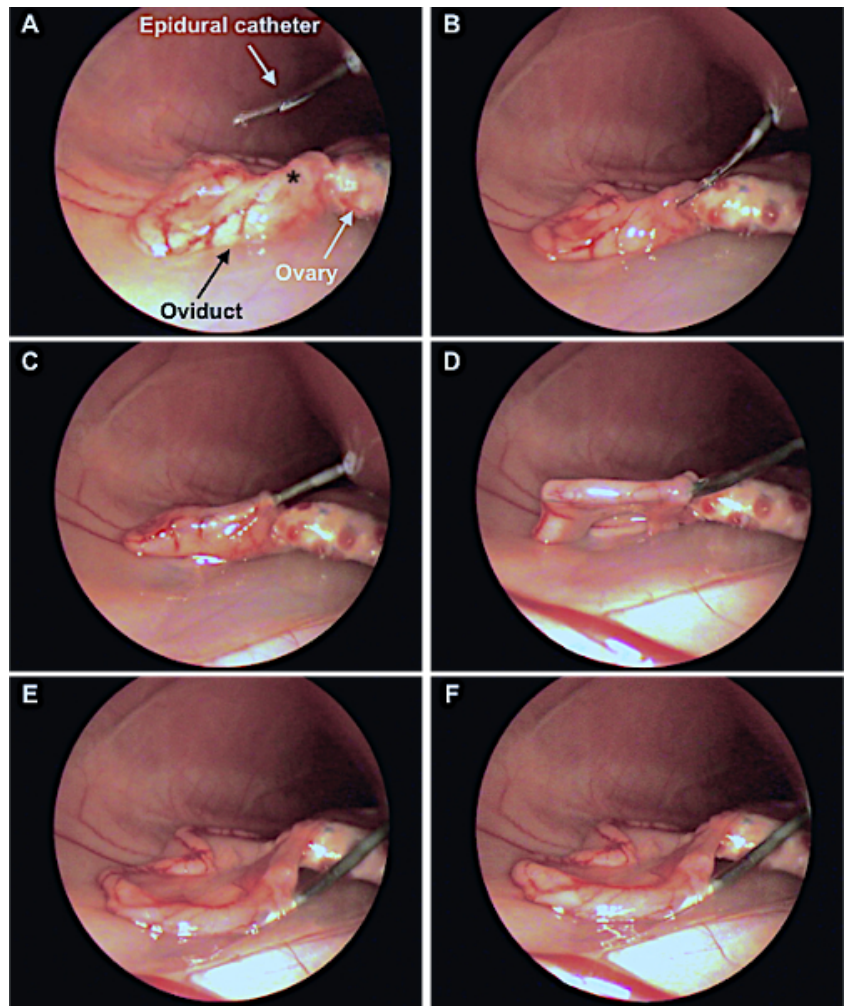

Figure 2: Laparoscopic embryo transfer assisted by laparoscopy (Internal view). A: Insertion of the catheter through the epidural needle into the abdominal zone. Asterisk indicates the infundibulum. B, C, D: The catheter loaded with the embryos is inserted into ampulla region across the infundibulum. E, F: Release of the embryos, confirmed by the visualization of a swollen oviduct. This figure has been adapted from Marco-Jiménez et al. ${ }^{38}$. Please click here to view a larger version of this figure.

\section{Embryo Vitrification and Warming}

1. Perform all the manipulations at room temperature (around $22{ }^{\circ} \mathrm{C}$ ) to reduce the vitrification solution toxicity at warmer temperatures. NOTE: Embryos can be moved using 0.1-2 $\mu \mathrm{L}$ automatic pipette in this protocol, but other similar devices to move the embryos dragging the minimum volume can be suitable.

2. Vitrify the embryos in a two-step addition procedure:

1. Place the embryos for 2 minutes in equilibrating solution consisting of $10 \%(\mathrm{v} / \mathrm{v})$ ethylene glycol and $10 \%(\mathrm{v} / \mathrm{v})$ dimethyl sulfoxide dissolved in BM.

2. Move the embryos (from step 2.2.1) for 1 minute into vitrification solution consisting of $20 \%(\mathrm{v} / \mathrm{v}$ ) ethylene glycol and $20 \%$ (v/v) dimethyl sulfoxide dissolved in BM. 
3. Load the embryos into a $125 \mu \mathrm{L}$ plastic ministraw (which contains one closed end with a cotton plug and one open extreme). The process is schematized in Figure 3.

1. Couple the closed end of $0.125 \mu \mathrm{L}$ ministraw with the appropriate $1 \mathrm{~mL}$ syringe.

2. Aspirate BM until $1 / 3$ of the straw length, following by a small air bubble.

3. Aspirate the embryos in a volume of $40 \mu \mathrm{L}$ of vitrification solution, followed by another small air bubble.

4. Aspirate BM until the first liquid fraction (step 2.3.2) reaches the cotton.

5. Close the open end with a straw plug.

4. Perform step 2.2.2 while step 2.3 is being done to ensure that no more than one-minute elapses, which would be toxic to embryos

5. Plunge the ministraw directly into liquid nitrogen to achieve vitrification.

6. Store the ministraw in a dewar for nitrogen for the desired time.

7. Thaw the embryos in a single step.

1. Place the ministraw horizontally $10 \mathrm{~cm}$ from liquid nitrogen vapour for $20-30 \mathrm{~s}$.

2. When the crystallization process begins inside the ministraw, immerse the ministraw in a water bath at $25^{\circ} \mathrm{C}$ for $10-15 \mathrm{~s}$.

3. Remove the ministraw plug and cut the cotton plug.

4. With a coupled syringe, expel all the ministraw content into a plate containing $0.33 \mathrm{M}$ sucrose solution at $25^{\circ} \mathrm{C}$ in $\mathrm{BM}$ for 5 minutes NOTE: This step must be done quickly in order to reduce embryo exposure to the vitrification solution.

5. Move the embryos to a new plate containing BM solution for another $5 \mathrm{~min}$.

6. Consider only non-damaged embryos (with intact mucin coat and zona pellucida) to continue with the ET. NOTE: Take into account that in thawed embryos, asynchronous transfers (e.g., 60-62 $\mathrm{h}$ in morula transfers) may improve the results by allowing a resynchronization between the embryo and the maternal endometrium.

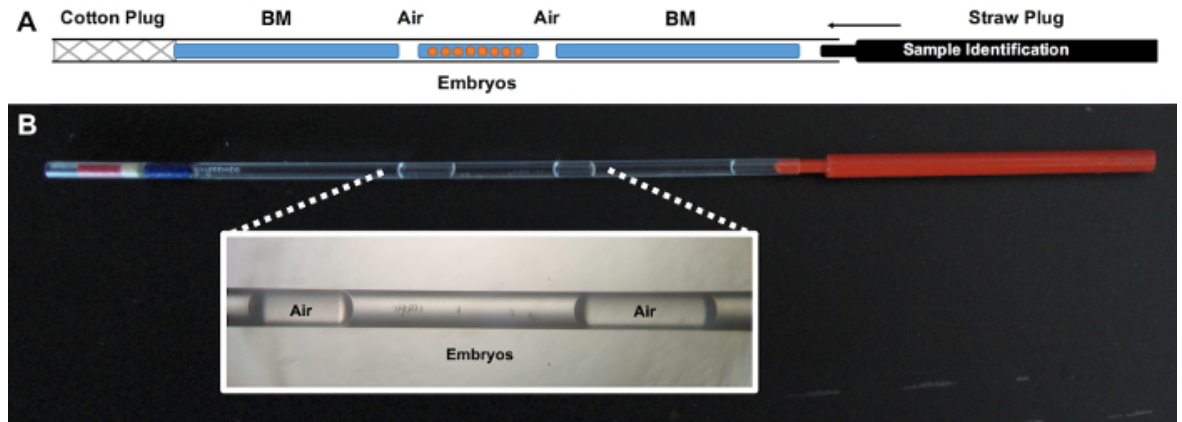

Figure 3: Schematization of correctly loaded straw. A) BM refers to the embryo manipulating media employed during vitrification. Embryos must be loaded in vitrification solution. B) Macroscopic appearance of the loaded straw with a magnified detail of the embryo position. This largevolume device allows us to vitrify large number of embryos, unlike minimum volume devices. Furthermore, the handling of this device is easier compared with minimum volume devices, while the results are similar in rabbits ${ }^{41}$. Please click here to view a larger version of this figure.

\section{Representative Results}

Minimally invasive laparoscopic transfer of fresh or vitrified embryos places the rabbit among the best model animals for reproductive studies. Table 1 shows the results of fresh ET at different developmental stages (Figure 4) of transferred embryos. The survival rate at birth (percentage of embryos resulting in a pup) proved the efficacy of the laparoscopic technique described in this paper. The higher values were achieved when the ET was performed with embryos in the morula stage, either early or compact morulae. Based on these results, we performed a second experiment to demonstrate the survival rate after vitrification of these embryos. Thus, in Table 2 we show the results obtained after transferring vitrified rabbit morulae recovered at the same time, differentiating between those embryos that had reached a good degree of compaction or not. The survival rate at birth was different between the different embryo stages, being higher in compacted morulae. Therefore, laparoscopic embryo transfer is a reliable technique to transfer fresh and vitrified embryos in rabbits 


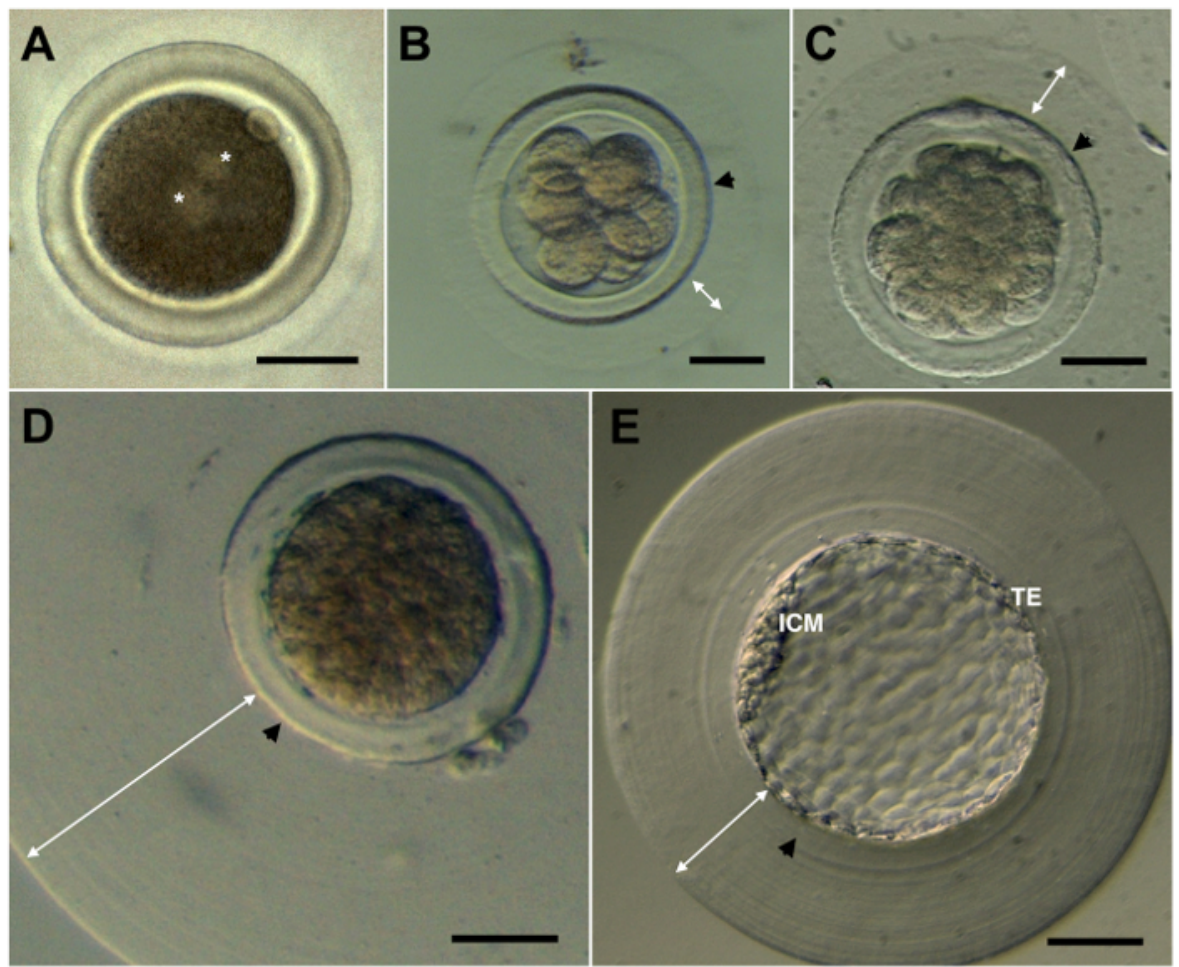

Figure 4: Rabbit embryos. A) Pronuclear. B) Eight cells. C) Early morula. D) Compact morula. E) Blastocyst. Asterisk indicates the two pronuclei. Black arrows indicate the zona pellucida. White arrows indicate the mucin coat, which normally varies between embryos. ICM: Inner Cell Mass. TE: Trophectoderm. Scale bar: $50 \mu \mathrm{m}$. Please click here to view a larger version of this figure.

\begin{tabular}{|l|l|l|l|l|l|l|}
\hline $\begin{array}{l}\text { Developmental } \\
\text { stage }\end{array}$ & Embryos & Recipients & Place of transfer & $\begin{array}{l}\text { Pregnancy rate } \\
(\%)\end{array}$ & $\begin{array}{l}\text { Implantation rate } \\
(\%)\end{array}$ & $\begin{array}{l}\text { Survival rate at } \\
\text { birth }(\%)^{2}\end{array}$ \\
\hline Pronuclear & 78 & 7 & Oviduct & $7(100)$ & $50(64.0)^{\mathrm{b}}$ & $34(43.6)^{\mathrm{b}}$ \\
\hline $\mathbf{8}$ cells & 81 & 7 & Oviduct & $7(100)$ & $60(74.1)^{\mathrm{b}}$ & $53(65.4)^{\mathrm{a}}$ \\
\hline Early morula & 81 & 7 & Oviduct & $7(100)$ & $80(98.8)^{\mathrm{a}}$ & $60(74.1)^{\mathrm{a}}$ \\
\hline Compact morula & 80 & 7 & Oviduct & $7(100)$ & $80(100)^{\mathrm{a}}$ & $58(72.5)^{\mathrm{a}}$ \\
\hline Blastocyst & 80 & 7 & Uterus & $7(100)$ & $73(91.3)^{\mathrm{a}}$ & $38(47.5)^{\mathrm{b}}$ \\
\hline
\end{tabular}

Table 1. Efficiency of fresh rabbit embryo transfer (in vivo derived) by laparoscopy. ${ }^{1}$ Different embryos were recovered at $18-20 \mathrm{~h}$ (pronuclear), 36-38h (8 cells), 60-62h (early morula), 70-72h (compact morula) and 80-82h (blastocyst) after mating. Compact (>32 cells) and non-compact morulae $\left(\approx 32\right.$ cells) can be founded at $70-72 \mathrm{~h}$, but only compact morulae were transferred. ${ }^{2}$ Survival rate at birth from recipient pregnant does. ${ }^{a, b}$ Values with different superscripts are statistically different $(P<0.001)$.

\begin{tabular}{|l|l|l|l|l|}
\hline Developmental stage & Transferred embryos & Recipients & Pregnancy rate (\%) & Survival rate at birth (\%) \\
\hline Non- compacted & 135 & 10 & $9(90)$ & $62(45.9)^{\mathrm{b}}$ \\
\hline Compacted & 150 & 10 & $10(100.0)$ & $98(65.3)^{\mathrm{a}}$ \\
\hline TOTAL & 285 & 20 & $19(95)$ & $160(56.1)$ \\
\hline
\end{tabular}

Table 2. Viability of non-compacted vs compact vitrified morula. ${ }^{a, b}$ Values with different superscripts are statistically different $(P<0.001)$.

${ }^{1}$ Survival rate at birth from recipient pregnant does. Embryos were recovered at the same time $(70-72 \mathrm{~h})$ and were distinguished into compact (>32 cells) and non-compact morulae ( $\approx 32$ cells).

\section{Discussion}

Since the first documented live birth case from transferred embryos ${ }^{9}$, this technique and the rabbit species have become crucial in reproductive studies. Besides, embryo research studies involving manipulation, production, cryopreservation, etc. require as a last step the evaluation of embryo capacity to generate healthy full-term offspring. Therefore, embryo transfer technique is indispensable ${ }^{13,28}$. Over the years, the surgical methods initially employed to transfer embryos into the maternal uterus have gradually been replaced by less invasive methods in the vast majority of species ${ }^{13,14,15,21,27,29,30}$. However, in rabbits, intraoviductal ET in early embryo stages of development and in vitro produced embryos becomes unavoidable to ensure a similar result to natural conditions. In rabbits, intraoviductal mucin coat is a crucial factor allowing embryo 
implantation, as it takes place after the remodelling of the embryonic coatings during blastocyst expansion in the uterine horns. However, mucin coat deposition is limited to the oviduct for 3 days following ovulation, and the molecular mechanisms of coat material deposition are largely unknown $^{31}$. For these reasons, it is known that in vitro-developed blastocysts did not survive when transferred to the uterus ${ }^{32,33,34}$, and embryos with a damaged mucin coat have a lower survival rate ${ }^{35}$. Likewise, groups that reported a transcervical embryo transfer in rabbits resulted in very low live born rates ${ }^{11,26}$. Here, we present a minimally invasive technique, adapted from Besenfelder and Brem ${ }^{18}$, to transfer embryos with successful birth rates. According to the results in Table 1, the morula stage in rabbit embryos was the best embryonic stage to achieve a high survival rate at birth. One possible explanation is the greater sensitivity to manipulation of the earliest stages. Interestingly, the success rate increases as the embryonic stage progresses, possibly due to the greater exposure of the embryo to oviductal secretions prior to its recovery. But when embryos reach the blastocyst stage and are place-concordant transferred to the uterus, the values decrease drastically. Not excluding what has been said, a possible explanation could be that the embryos transferred into the oviduct can restore the possible damage generated in the mucin layer during embryo manipulation. Therefore, blastocysts transferred into the uterus would be deprived of this mechanism, which could compromise their implantation capacity.

The technique is performed using a single port instrument $(5 \mathrm{~mm}$ endoscope trocar), with slight, brief manipulation. Therefore, the5-mm endoscope trocar incision does not require closure.Laparoscopic technique benefits include decreased postoperative pain, quicker return to normal activity, and fewer postoperative complications. In addition, endoscopic procedures induce fewer abdominal adhesions and allow a better immune response by the recipient compared with open surgery ${ }^{21,36,37}$. Accumulating evidence from our lab has demonstrated the effectiveness of this ET procedure in the rabbit model. Thus, in the last five years a total of 3,909 embryos $(1,335$ fresh and 2,574 vitrified embryos) were transferred through the procedure described in the present manuscript. As a result of this technique, the offspring rates of fresh and vitrified transfer embryos were $62.9 \%$ and $42.5 \%$, respectively ${ }^{38,39,40,41,42,43,44,45,46,47}$. Many studies are all based on this technique: Marco-Jiménez et al. $^{38,39,40,41}$, Vicente et al. ${ }^{42}$, Viudes-de-Castro et al. ${ }^{43}$, Saenz-de-Juano et al. ${ }^{44,45,47}$, Lavara et al. ${ }^{46}$.

Practical recommendations for carrying out this technique are described below. In embryo culture experiments, it is also advisable to use a new catheter for embryo transfer instead of the one used to move the embryos between the culture media and manipulation media. This avoids transfer of mineral oil and ensures an optimal flow. During ET it is important to minimize handling of the reproductive tract, as excessive manipulation of the oviduct could result in adhesions. If the oviduct is twisted, employ the epidural syringe to try to position it correctly, not the catheter, as it contains the embryos and the mechanical manipulation could cause their loss. Once the catheter passes through the oviduct, it slides easily. If it does not, the catheter may have deviated. Once inside the oviduct, if the media does not flow, move the catheter out slightly and try to reinsert it again. If it still does not flow, the catheter is clogged. Remove it from the oviduct and release the content into a dish with a clean medium. Then, reload the embryos into another catheter and try to reinsert it into the oviduct again. Delivery usually takes places $28-30$ days after morula transfer.

In addition, there is evidence indicating that the embryo developmental stage can be more advanced than the uterine environment in pseudopregnant females, but not the opposite. Specifically, embryos have the ability to wait for the favorable womb environment, but the womb environment cannot wait for the embryos at the right stage for implantation ${ }^{10}$. With regard to vitrified embryos, after a short/Long-term storage it is possible to synchronize the developmental stage of the embryo with the corresponding favorable womb environment. Furthermore, if the embryo donor is also the embryo recipient, the detrimental effects of superovulation on the endometrium can be bypassed by using the vitrification technique and transferring the embryos in a subsequent cycle ${ }^{48}$. In rabbits, vitrified embryos transferred into oviducts of recipients induced to ovulate $60-62 \mathrm{~h}$ beforehand (asynchrony) is a highly efficient technique ${ }^{44,49}$. Related with this, it has been suggested that the oviductal embryo transition during 10-12 h could explain the beneficial effects in the restoration of cell physiology and replacement of dead cells, and probably repair the damage induced in mucin coat during embryo manipulation. Besides, vitrified embryos present a delay in development, as they have been metabolically suspended during the storage. Therefore, transfer of cryopreserved embryos into asynchronous recipients allows the embryo to reactivate its metabolic activity and thus the embryo stage of development is synchronized with the womb environment. Instead, if cryopreserved embryos are transferred into synchronic receptors, the cross-talk between the mother and the embryo hinders the onset of a successful pregnancy. In rabbit, the highest survival rate has been obtained after intraoviductal transfer of cryopreserved morulae ${ }^{49}$. Our data are consistent with this report, although the morula stage exhibits different survival rates following cryopreservation depending on their degree of compaction at 70-72h (Table 2). Here, compacted morulae showed higher survival rates at birth in comparison to non-compacted morulae, which was in concordance with previous reports showing that every stage of development had its own mechanism relative to the permeation of cryoprotectants and the extent of dehydration during the addition of the cryopreservation solution ${ }^{50}$. Underlying these techniques, we have demonstrated that a combination of vitrification and intraoviductal embryo transfer is a successful strategy to re-establish rabbit populations after 15 years of storage in liquid nitrogen, without adverse effect on their post-thaw survival and live birth ${ }^{51}$.

The following details should be taken into account to successfully perform this technique. It is important to bear in mind that the increasing density of the consecutive mediums used for vitrification (DPBS, equilibration solution, vitrification solution) could induce embryo contraction due to progressive embryo dehydration. However, its normal appearance is recovered when the embryo is equilibrated with the medium. Furthermore, when the embryo is moved between increasing density media, it tends to move to the surface of the media due to density movements. To avoid embryo loss and ensure the time of vitrification, it is recommendable to perform the vitrification in small drops of the media that will keep the embryo in place.

In conclusion, here we describe both an ET technique and an embryo vitrification method that facilitate future studies which use rabbits as a model. Based on the close phylogenetic distance between rabbits and humans, the use of this model could provide results easily transferable to human clinical medicine. In addition, our method offers some hygienic and economic advantages, conforming to the concept of the 3 Rs of animal welfare (replacement, reduction and refinement), while maintaining the goal of improving humane treatment of experimental animals.

\section{Disclosures}

The authors have nothing to disclose. 


\section{Acknowledgments}

This work was supported by funds from the Ministry of Economy and Competitiveness of Spain Research Programme (AGL2014-53405-C2-1P) and Generalitat Valenciana Research Programme (Prometeoll 2014/036). English text version revised by N. Macowan English Language Service.

\section{References}

1. Chen, M., Heilbronn, L.K. The health outcomes of human offspring conceived by assisted reproductive technologies (ART). Journal of Developmental Origins of Health and Disease. 8 (4), 388-402 (2017).

2. Lavara, R., Baselga, M., Marco-Jiménez, F., Vicente, J.S. Embryo vitrification in rabbits: Consequences for progeny growth. Theriogenology. 84 (5), 674-80 (2015).

3. Sirard, M.A. The influence of in vitro fertilization and embryo culture on the embryo epigenetic constituents and the possible consequences in the bovine model. Journal of Developmental Origins of Health and Disease. 8 (4), 411-417 (2017).

4. Feuer, S.K., Rinaudo, P.F. Physiological, metabolic and transcriptional postnatal phenotypes of in vitro fertilization (IVF) in the mouse. Journal of Developmental Origins of Health and Disease. 8 (4), 403-410 (2017).

5. Jiang, Z., et al. Genetic and epigenetic risks of assisted reproduction. Best Practice \& Research: Clinical Obstetrics \& Gynaecology. 44, 90-104 (2017).

6. Fleming, T.P, Velazquez, M.A., Eckert, J.J. Embryos, DOHaD and David Barker. Journal of Developmental Origins of Health and Disease. 6 (5), 377-83 (2015).

7. Sparks, A.E. Human embryo cryopreservation-methods, timing, and other considerations for optimizing an embryo cryopreservation program. Seminars in Reproductive Medicine. 33 (2), 128-44 (2015).

8. Swain, J.E. Optimal human embryo culture. Seminars in Reproductive Medicine. 33 (2), 103-17 (2015).

9. Heape, W. Preliminary note on the transplantation and growth of mammalian ova within a uterine foster-mother. Proceedings of the Royal Society of London B: Biological Sciences. 48, 457-9 (1890).

10. Bermejo-Alvarez, P., Park, K.E., Telugu, B.P. Utero-tubal embryo transfer and vasectomy in the mouse model. Journal of Visualized Experiments. 84, e51214 (2014).

11. Kidder, J.D., Roberts, P.J., Simkin, M.E., Foote, R.H., Richmond, M.E. Nonsurgical collection and nonsurgical transfer of preimplantation embryos in the domestic rabbit (Oryctolagus cuniculus) and domestic ferret (Mustela putorius furo). Journal of Reproduction and Fertility. 116 (2), 235-42 (1999).

12. Tıras, B., Cenksoy, P.O. Practice of embryo transfer: recommendations during and after. Seminars in Reproductive Medicine. 32 (4), $291-6$ (2014).

13. Cui, L., et al. Transcervical embryo transfer in mice. Journal of the American Association for Laboratory Animal Science. 53 (3), $228-31$ (2014).

14. Moreno-Moya, J.M., et al. Complete method to obtain, culture, and transfer mouse blastocysts nonsurgically to study implantation and development. Fertility and Sterility. 101 (3), e13 (2014).

15. Hasler, J.F. Forty years of embryo transfer in cattle: a review focusing on the journal Theriogenology, the growth of the industry in North America, and personal reminisces. Theriogenology. 81 (1), 152-69 (2014).

16. Bauer, C. The baboon (Papio sp.) as a model for female reproduction studies. Contraception. 92 (2), 120-3 (2015).

17. Martinez, E.A., et al. Nonsurgical deep uterine transfer of vitrified, in vivo-derived, porcine embryos is as effective as the default surgical approach. Science Reports. 5, 10587 (2015).

18. Besenfelder, U., Brem, G. Laparoscopic embryo transfer in rabbits. Journal of Reproduction and Fertility . 99, 53-56 (1993).

19. Besenfelder, U., Mödl, J., Müller, M., Brem, G. Endoscopic embryo collection and embryo transfer into the oviduct and the uterus of pigs. Theriogenology. 47 (5), 1051-60 (1997).

20. Besenfelder, U., Havlicek, V., Kuzmany, A., Brem, G. Endoscopic approaches to manage in vitro and in vivo embryo development: use of the bovine oviduct. Theriogenology. 73 (6), 768-76 (2010).

21. Fonseca, J.F., et al. Nonsurgical embryo recovery and transfer in sheep and goats. Theriogenology. 86 (1), 144-51 (2016).

22. Denker, H.W. Structural dynamics and function of early embryonic coats. Cells Tissues Organs. 166, 180-207 (2000).

23. Marco-Jiménez, F., López-Bejar, M. Detection of glycosylated proteins in rabbit oviductal isthmus and uterine endometrium during early embryo development. Reproduction in Domestic Animals. 48 (6), 967-73 (2013).

24. Ménézo, Y.J., Hérubel, F. Mouse and bovine models for human IVF. Reproductive BioMedicine Online. 4 (2), 170-5 (2002).

25. Fischer, B., Chavatte-Palmer, P., Viebahn, C., Navarrete Santos, A., Duranthon, V. Rabbit as a reproductive model for human health. Reproduction. 144 (1), 1-10 (2012).

26. Besenfelder, U., Strouhal, C., Brem, G. A method for endoscopic embryo collection and transfer in the rabbit. Zentralb/ Veterinarmed A. 45 (9), 577-9 (1998).

27. Daniel, N., Renard, J.P. Embryo transfer in rabbits. Cold Spring Harbor Protocols. 2010 (1) (2010).

28. Saenz-de-Juano, M.D., et al. Vitrification alters rabbit foetal placenta at transcriptomic and proteomic level. Reproduction. 147 (6), 789-801 (2014).

29. Green, M., Bass, S., Spear, B. A device for the simple and rapid transcervical transfer of mouse embryos eliminates the need for surgery and potential post-operative complications. Biotechniques. 47 (5), 919-24 (2009).

30. Duan, X., Li, Y., Di, K., Huang, Y., Li, X. A nonsurgical embryo transfer technique in mice. Sheng Wu Gong Cheng Xue Bao. 32 (4), $440-446$ (2016).

31. Denker, H.W., Gerdes, H.J. The dynamic structure of rabbit blastocyst coverings. I: transformation during regular preimplan- tation development. Anatomy and Embryology. 157, 15-34 (1979).

32. Seidel, G.E., Bowen, R.A., Kane, M.T. In vitro fertilization, culture and transfer of rabbit ova. Fertility and Sterility. 27: 861-870 (1976).

33. Binkerd, P.E., Anderson, G.B. Transfer of cultured rabbit embryos. Gamete Research. 2, 65-73 (1979). 
34. Murakami, H., Imai, H. Successful implantation of in vitro cultured rabbit embryos after uterine transfer: a role for mucin. Molecular Reproduction and Development . 43, 167-170 (1996).

35. Techakumphu, M., Wintenberger-Torrèsa, S., Sevelleca, C., Ménézo, Y. Survival of rabbit embryos after culture or culture/freezing. Animal Reproduction Science. 13 (3), 221-228 (1987).

36. Gitzelmann, C.A, et al. Cell-mediated immune response is better preserved by laparoscopy than laparotomy. Surgery. 127 (1), 65-71(2000).

37. Huang, S.G., Li, Y.P., Zhang, Q., Redmond, H.P., Wang, J.H., Wang, J. Laparotomy and laparoscopy diversely affect macrophage-associated antimicrobial activity in a murine model. BMC Immunology. 14, 27 (2013).

38. Marco-Jiménez, F., Jiménez-Trigos, E., Almela-Miralles, V., Vicente, J.S. Development of Cheaper Embryo Vitrification Device Using the Minimum Volume Method. Public Library of Science One. 11 (2), e0148661 (2016).

39. Marco-Jiménez, F., Jiménez-Trigos, E., Lavara, R., Vicente, J.S. Generation of live offspring from vitrified embryos with synthetic polymers supercool X-1000 and Supercool Z-1000. CryoLetters. 35, 286-292 (2014).

40. Marco-Jiménez, F., Jiménez-Trigos, E., Lavara, R., Vicente, J.S. Use of cyclodextrins to increase cytoplasmic cholesterol in rabbit embryos and their impact on live KITs derived from vitrified embryos. Cryoletters. 35, 320-326 (2014).

41. Marco-Jiménez, F., Lavara, R., Jiménez-Trigos, E., Vicente, J.S. In vivo development of vitrified rabbit embryos: Effects of vitrification device, recipient genotype, and asynchrony. Theriogenology. 79 (7), 1124-9 (2013).

42. Vicente, J.S, et al. Rabbit morula vitrification reduces early foetal growth and increases losses throughout gestation. Cryobiology. 67, 321-326 (2013).

43. Viudes-de-Castro, M.P., Marco-Jiménez, F., Cedano-Castro, J.I., Vicente, J.S. Effect of corifollitropin alfa supplemented with or without Lh on ovarian stimulation and embryo viability in rabbit. Theriogenology. 98, 68-74 (2017).

44. Saenz-de-Juano, M.D., et al. Vitrification alters at transcriptomic and proteomic level rabbit foetal placenta. Reproduction. 147, 789-801 (2014).

45. Saenz-de-Juano, M.D, Marco-Jimenez, F., Viudes-de-Castro, M.P., Lavara, R., Vicente, J.S. Direct comparison of the effects of slow freezing and vitrification on late blastocyst gene expression, development, implantation and offspring of rabbit morulae. Reproduction in Domestic Animals. 49, 505-511 (2014).

46. Lavara, R., Baselga, M., Marco-Jiménez, F., Vicente, J.S. Long-term and transgenerational effects of cryopreservation on rabbit embryos. Theriogenology. 81, 988-992 (2014).

47. Saenz-de-Juano, M.D., Marco-Jiménez, F., Vicente, J.S. Embryo transfer manipulation cause gene expression variation in blastocysts that disrupt implantation and offspring rates at birth in rabbit. European Journal of Obstetrics \& Gynecology and Reproductive Biology. 207, 50-55 (2016).

48. Roque, M., Valle, M., Kostolias, A., Sampaio, M., Geber, S. Freeze-all cycle in reproductive medicine: current perspectives. JBRA Assisted Reproduction. 21 (1), 49-53 (2017).

49. Tsunoda, Y., Soma, T., Sugie, T. Effect of post-ovulatory age of recipient on survival of frozen-thawed rabbit morulae. Journal of Reproduction and Fertility. 65 (2), 483-7 (1982).

50. Vanderzwalmen, P., et al. Births after vitrification at morula and blastocyst stages: effect of artificial reduction of the blastocoelic cavity before vitrification. Human Reproduction. 17 (3), 744-51 (2002).

51. Lavara, R., Baselga, M., Vicente, J.S. Does storage time in LN2 influence survival and pregnancy outcome of vitrified rabbit embryos? Theriogenology. 76 (4), 652-7 (2011). 